\title{
Galactic disks and their evolution
}

\author{
A. V. Zasov* \\ Sternberg Astronomical Institute Lomonosov Moscow University \\ O. K. Sil'chenko \\ Sternberg Astronomical Institute Lomonosov Moscow University
}

\begin{abstract}
We consider the key problems related to measuring the mass of stellar disks and dark halos in galaxies and to explaining the observed properties of disks formed in massive dark halos.

Published in Uspekhi Fizicheskikh Nauk 180 (4) 434-439 (2010)

DOI: $10.3367 / U F N r .0180 .201004 i .0434$
\end{abstract}

Rotating stellar-gas disks are the main structural elements of the majority of the observed galaxies. They contain mostly baryonic matter: stars and the diffuse interstellar medium (in the latter, cold gas dominates). Disks have a large angular momentum and rotate such that the local angular velocity decreases along the radius. The maximum rotational velocity of the disk depends on the total mass (luminosity) of the galaxy and is usually $100-300 \mathrm{~km} / \mathrm{s}$, which corresponds to the orbital period $200-300 \mathrm{Myr}$.

Disks of galaxies are inhomogeneous structures. They contain stars with different masses and ages, and the youngest stars are located near the equatorial plane of the disk: there, on the bottom of the disk gravitational potential well, the interstellar gas is stored. But because the entire disks are old structures, their spectra are dominated by very old stars with the age above $8 \mathrm{Gyr}$. The oldest stars form the so-called thick disk, which is two to three times thicker than the main stellar disk, but the mass of the thick disk is relatively small. In fact, the formation of disks in most galaxies has not yet been completed, because star formation is occurring (predominantly in the spiral arms) there even at present, but the observed rates of stellar population mass growth, except for rare cases, are very low, about 1-5 solar masses per year for an entire galaxy like our own.

The origin of galaxies and their disk formation mechanism remain unclear and are actively discussed in the literature. The very form of the disks clearly suggests that they were formed as a result of the evolution of a dissipative medium (gas), which had been losing its energy by radiation with angular momentum conservation, and the age of the disks suggests that they already existed in the very early times of modern galaxies and had a very intensive star formation rate at that time. Disk galaxies, which frequently have big star- forming regions that appear from large distances like a collection of individual bright spots, are indeed abundantly present among galaxies with redshifts $z>1$;

*zasov@sai.msu.ru we observe them at the time of their youth.

In modern galaxy formation theories, it is important that star formation in galaxies occurred in the gravitational field formed by dark matter, or the so-called hidden mass, which presently must form massive halos around galaxies extending far away from their visible parts. Numerical simulations showed that the role of the dark halo is decisive in both the disk formation and its later evolution. But it is not an easy task to decompose the observed mass of a galaxy into gas, stellar, and dark matter components. This problem can be solved for individual galaxies by improving upon methods of measuring the kinematical characteristics of the disk, on the one hand, and models of evolution of stellar population spectra, on the other hand.

The scientific boom that gave rise to an avalanche of studies of hidden mass inside galaxies started when observational data on galaxy disk rotational velocity curves $V(R)$ as a function of radius $\mathrm{R}$ became available for sufficiently large distances from the center. In the optical band, either classical diffraction spectrographs with a long slit, or scanning Fabry-Perot interferometers in a high order of interference are typically used. The interferometers do not have slits, and the Doppler shifts of spectral lines can be measured simultaneously at many thousands of points on the galactic disk; using a complicated mathematical data processing then allows recovering the two-dimensional radial velocity field and obtaining the rotational curve. In Russia, such observations have been carried out on the 6-meter telescope at the Special Astrophysical Observatory RAS. Radio observations in the emission lines of atomic hydrogen or molecules have a lower angular resolution than in the optical lines, while optical observations can be used to measure radial velocities of both gas and stars with a high angular resolution. Nevertheless, radio velocity curves in gas-rich galaxies are traced to much longer distances than the optical ones, sometimes reaching far beyond the visible limits of a galaxy, because gas disks are frequently much larger than stellar ones. It was found that rotational velocities at large distances from galactic centers do not decrease, as a rule, but become almost flat (come to a plateau), or even increase 
with $R$.

That the rotational velocity reaches a plateau is frequently considered if not the proof, at least a decisive argument in favor of the existence in galaxies of a dark halo with a mass comparable with or even exceeding that of visible matter. In fact, this is not precisely the case because the form of the rotational velocity curve, for some reason, does not automatically imply the presence of dark matter. A rotational curve of any form, increasing or decreasing, can be explained by the presence of only one disk, and it can reflect only the peculiarity of mass distribution inside it. We illustrate this with simple examples.

If the disk density along the radius were conserved or decreased very slowly, the rotational velocity of the disk would increase without bound as $\mathrm{R}$ increases, even in the absence of a halo. Of course, the surface density is not constant in real galactic disks; it quite rapidly decreases with the distance from the center. But there is no major problem explaining the plateau on the rotational curve. The classical example is the so-called Mestel disk. This is a thin axially symmetric disk whose surface density $\Sigma(R)$ decreases from the center to the periphery as $1 / R$. It can be shown theoretically that the circular velocity, which in the general case is determined by the radial gradient of the gravitational potential,

$$
V^{2}(R)=R \cdot \partial \Phi(R) / \partial R
$$

is independent of $R$ for the Mestel disk, and the rotational curve of the galaxy is a horizontal line from zero to infinity, such no dark halo is to be invoked! The mass of such a disk within any given radius $R$ is $M(R)=V^{2} R / G$, i.e., the same as for a spherically symmetric density distribution. In other words, it is impossible to distinguish the Mestel disk from a spherically symmetric galaxy using the form of the rotational velocity curve.

In that case, what indeed can be considered relevant to the presence of a hidden mass in disk galaxies, in particular, a massive dark halo? First, this is a discrepancy between the measured rotational velocity curve of the galactic disk (using both the form and the absolute value of velocities) and the expected curve calculated by assuming that the galaxy consists of only 'luminous' matter, i.e., of the directly observed components. Because most of the mass of the disk resides in stars, the brightness distribution of the stellar disk reflects its mass distribution, especially if the brightness is measured in the near-infrared spectral band, where emission from old stars dominates.

The brightness and hence the surface density in the broad distance range from the center decreases with $R$ exponentially as $I(R) \sim \exp \left(-R / R_{0}\right)\left(R_{0}\right.$ is the radial scale of the brightness), i.e., more rapidly than $1 / R$. This law results in a rotational

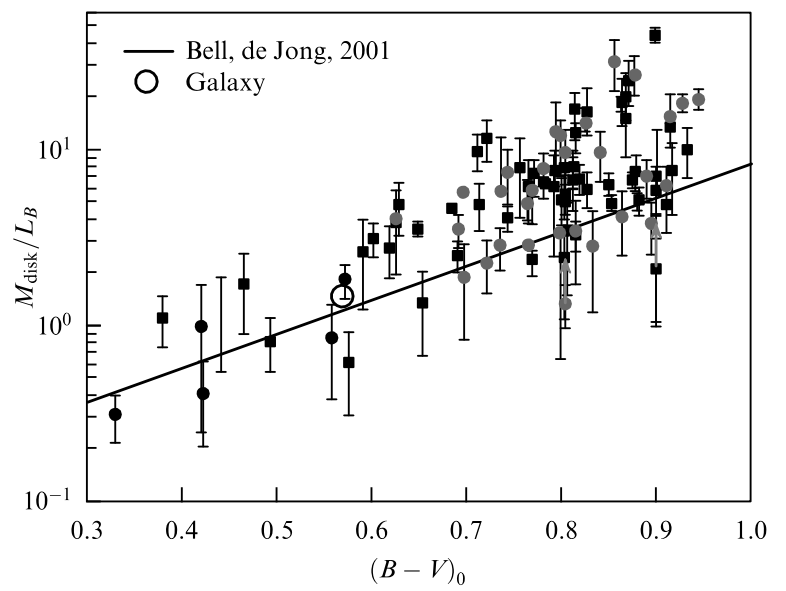

FIG. 1: Figure 1. The upper limit on the massluminosity ratio in the blue spectral range $M / L_{B}$ (in solar units) for galactic disks with different color indices, derived under the assumption of marginal disk stability [7]. The black circles show galaxies in pairs, the black squares mark isolated galaxies or members of groups, and the white circle stands for our Galaxy. The values of $M / L_{B}$ for galaxies with active star formation $\left((B-V)_{0}>0.7\right)$ are close to those expected from the photometrical model of stellar systems evolution (the straight line according to [3]), which suggests the absence of strong dynamical heating of their disks.

curve reaching a maximum at $R \approx 2 R_{0}$ and then slowly decreasing. However, the expected maximum is never observed in real galactic rotational curves. In a more rigorous approach, by adopting a certain mass-luminosity ratio $M / L$ for the stellar population of the disk (this ratio can be estimated from the observed color indices using stellar population models), the brightness distribution can be easily transformed into the density distribution along the disk (not necessarily exponential) and the expected rotational velocity of the galaxy due to its baryonic components can be calculated. In this way, the calculated rotation velocity curve typically passes lower than the observed one (at least in the outer parts of the disk). This allows concluding that dark mass significantly contributes to the total mass of the galaxy. This contribution is most substantial in galaxies with low surface brightness of the disk: in these galaxies, dark mass can exceed the baryonic mass by several times within the observed boundaries.

There are several other arguments in favor of high-mass dark halos in galaxies. Two main arguments follow from observations, although they are statistical. The first argument is that the integral masses of galaxies and galaxy systems measured by other means (using the relative velocities of the motion of satellites) are found to be much larger than their visible mass. For example, the data in $[1,2]$ show that the mean ratio 
of the total mass of galaxies in pairs to their total infrared K-luminosity $(2.2 \mathrm{~mm})$, derived for more than 500 pairs, is very high: around 11 solar units, and for groups of galaxies, two times as high. For compar- ison, models of the purely stellar population yield the value of $M / L_{K}$ below 1.5 [3]. Accounting for the mass of gas and the internal extinction only slightly increases this ratio. Consequently, in the region whose size exceeds the diameter of the galaxy and includes a pair or group of galaxies, the mass of dark matter exceeds the total mass of directly visible matter by many times.

The second argument is related to the stability condition of the disk with respect to gravitational perturbations. The disk is usually described as a massive strongly oblate stellar system in which oscillations can propagate and instabilities can develop on different scales. This inevitably leads to the dynamic heating of the disk up to a state with marginal stability under small perturbations. The higher is the radial velocity dispersion of stars constituting most of the disk mass and the faster it rotates and the lower its surface density is, the more stable the disk is. The critical (maximal) surface density at which the disk is still stable is determined both analytically (under several simplifying assumptions) and numerically. In the first approximation, the critical surface density is proportional to the radial velocity dispersion times the angular velocity of the disk at a given radius. Hence, after having obtained the rotational velocity curve and velocity dispersion of old stars of the disk from observations, it is possible to estimate the maximum admissible surface density of the disk and the corresponding $M / L$ ratio, and then to find the upper limit of its mass from the total disk luminosity.

The accuracy of this estimate for an individual galaxy is not very high, up to a factor of two, but data obtained for different galaxies allow to make some general conclusions. Specifically, it has been confirmed that within the optical limits of a galaxy, the dark halo mass is typically comparable to that of the disk and often exceeds it [4], which is compatible with results derived from the analysis of rotational velocity curves. The same conclusion can be obtained from the photometric estimates of the thickness of stellar disks observed edge-on [5, 6]. There is another intriguing fact: because galactic disks are subjected to gravitational perturbations from both neighboring galaxies and massive dark halos (see below), it follows that, apparently, gravitational perturbations must have dynamically heated up the disk above the stability limit. Then the $M / L$ ratios calculated under the assumption of marginally stable disks (Fig. 1) exceed the values derived from the color index of photometrical models of the evolution of galactic stellar disks (the straight line in Fig. 1). It turns out that such overheated systems do exist, but only some fraction of galaxies, predominantly with high color indices

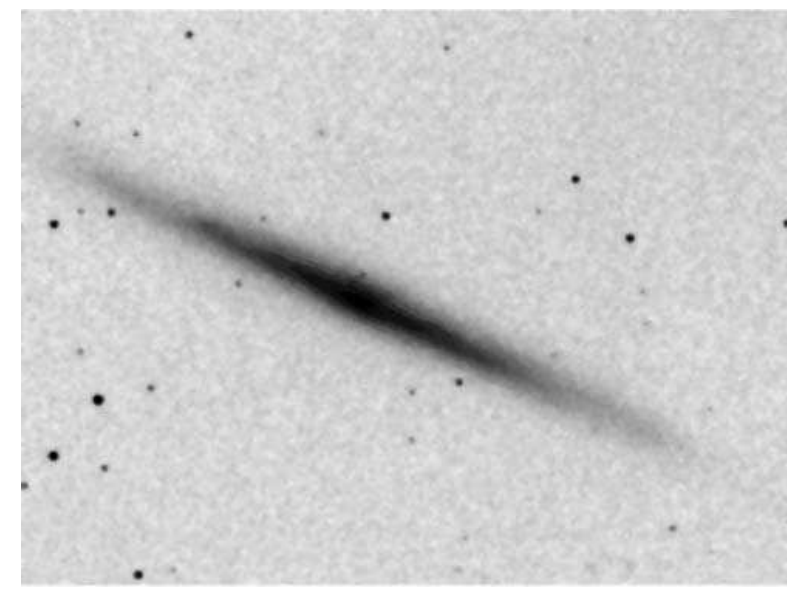

FIG. 2: Figure 2. NGC 5907, an example of a galaxy with a thin disk observed edge-on (2MASS (Two Micron All-Sky Survey), $2 \mathrm{mkm}$, near infrared).

(corrected for the disk inclination to the line of sight) $(B-V)_{0}>0.7$ relate to them. Such a color index corresponds to evolved disks whose luminosities are dominated by an old stellar population. In those galaxies, the star formation is very weak or totally absent; many of them are lenticular systems that contain almost no cold interstellar gas. Significantly 'overheated' disks are frequently observed in galaxies in pairs (circles in Fig. 1), obviously because they can experience a stronger gravitational perturbation from the companion. But among galaxies with 'overheated' disks, there are galaxies without close companions, and the increase in the velocity dispersion of their stars can then be due to the merging of the companions that are no longer observed as individual galaxies. But most important is that many lenticular and most spiral galaxies had no significant dynamical evolution over the several billion years of their lives, and their disks are kept weakly 'heated' in a state close to the marginal stable one. The presence of galaxies with enigmatically thin stellar disks points to the same fact. These galaxies show low vertical velocity dispersion with respect to the rotational velocity and are frequently found among galaxies observed edge-on (Fig. 2).

Yet another argument favoring the existence of dark halos and dark matter in the Universe in general has a rather theoretical character: it is impossible to calculate the physical picture of galaxy formation within the standard theory of the expanding universe without assuming that most matter in the Universe is a nonbaryonic dark matter. The proper gravity of baryonic matter, which amounts to four percent of the critical density of the Universe, is far too insufficient to explain how minuscule primordial fluctuations could grow in a short time to form the observed galaxies and their systems. 
In the framework of the so-called standard cosmological model, galaxies appear as a result of hierarchical clustering of numerous dark matter units (subhalos) in gravitational fields in which the primordial gas is concentrated, cools down, and then forms stellar galaxies. Later on, a long evolutionary path of the galaxy begins: the structure of the galaxy, the content and chemical abundance of gas and stars, and star formation rate can significantly change over several billion years. There are numerous problems from the theoretical standpoint: the role of processes such as the interaction of galaxies between themselves and with the intergalactic medium, galactic mergers, the activity of galactic nuclei, gas ejection from galaxies, and gas accretion on galactic disks that is capable of maintaining the current star formation rate for a long time remains unclear. In all these cases, the relative mass of dark matter in galaxies plays either a significant or a decisive role.

Numerical modeling of the galaxy formation process from dark matter and baryonic matter allowed finding, at least on the qualitative level, an explanation for the observed large-scale structure of the Universe and distribution of galactic mass. The galactic mass-size dependence [8] or mass concentration within the central kiloparsec, which is much lower than that predicted by numerical models (the so-called central cusp problem), are explained much more poorly. But one of the most relevant present-day problems of the hierarchical clustering model is the presence of a large number of purely disk galaxies in the nearby Universe (see, e.g., the statistics of the APM (Automated Plate Measuring) survey aimed at morphological classification of galaxies from their images [9]), i.e., galaxies without a significant central spheroid component, with only thin stellar disks. In many spiral galaxies, including our own, these thin stellar disks are also very old: the age of the oldest open clusters of our thin disk approaches 8-9 Gyr [10], and this means that starting from the time corresponding to the redshift $z=1$, our Galaxy has not been seriously 'disturbed'.

The above proximity of the velocity dispersion of the disks of many galaxies to the minimum value required by gravitational stability, as well as the small velocity dispersion of disk stars in our Galaxy, also suggest the absence of strong dynamical heating of many galactic disks (see, e.g., [11]). This directly contradicts the hierarchical concept that predicts permanent galactic mergings during the evolution of the Universe. When small halos merge to produce a $10^{12}$ solarmass halo (as in our Galaxy), the merged fragments preserve their identity inside the big halo for a long time. For example, recent GHALO (Galactic Halo) calculations [12] numerically modeling a small volume about $400 \mathrm{kpc}$ in size with high spatial resolution have identified up to a hundred thousand 'sub- halos' with continuous mass spectrum inside our dark halo. Dark matter clumps move inside the large halo on elongated orbits and inevitably cross the baryonic galactic disk. This 'bombardment' heats up the stellar disk, which gradually thickens due to an increase in the vertical velocity of stars. Moreover, dark matter clumps inside which stars emerged (dwarf satellites of our Galaxy) lose energy in encounters due to dynamical friction and finally fall on the disk to merge with it. According to recent calculations [13], a typical model galaxy similar to the Milky Way had to undergo about six 'minor' mergers during the last $8 \mathrm{Gyr}$ (the time corresponding to $z=1$ ), one of the mergers being with a satellite whose mass is about $10 \%$ of the mass of our Galaxy, which would thicken its disk by several times. In other words, thin stellar disks do not survive on a timescale of several billion years if they are plunged into dark matter halos as predicted by the theory; nevertheless, most nearby galaxies do have thin disks. This contradiction between theory and observations has not been resolved yet.

The key feature of the evolution of galactic disks is the permanent accretion of gas from outside, 'feeding' the star formation. The need for external gas accretion at a rate roughly comparable with that of star formation follows from many observational facts. In particular, the scenario of chemical evolution of the disk of our Galaxy cannot be built without considering an appreciable accretion of gas from outside (see, e.g., [14]). The star formation rate in the Galaxy disk over the last $9-10$ Gyr is nearly constant averaged over $1-2$ Gyr), which indirectly points to permanent gas accretion. Because stars synthesize all elements heavier than beryllium in the course of their evolution, the last generation of stars must be much more metalabundant than 8-10 Gyr old. However, no significant anticorrelation of of metallicity with age is observed [15]. Moreover, there is the so-called G-dwarf problem: in a galaxy disk, stars of spectral class $\mathrm{G}$ with a mass around one solar mass or slightly lighter, among which stars of all ages are present, have almost the same metallicity within measurement errors [16]. It seems that the chemical evolution in the disk of our Galaxy over the last 8-10 Gyr 'did not go forth,' although nuclear reactions in the stars have undoubtedly occurred. This problem is resolved by introducing accretion from outside, i.e., by assuming that gas with minimal (and even better, zero) metal abundance has fallen onto the disk: such a low-metallicity gas 'dilutes' the gas enriched by synthesized heavy elements and sustains the mean metallicity of the interstellar medium at a roughly constant level.

In spiral galaxy disks, including our own, there is a notable 'metallicity gradient': the mean heavyelement abundance in both stars and gas is higher at the center of the disk and decreases toward the 
disk periphery. Qualitatively, this is clear: in central parts of galaxies, star formation has already exhausted all the gas, i.e., was very effective, and on the periphery, a large amount of fresh gas remains, i.e., star formation has occurred very slowly. Naturally, star formation is then more effective at the disk center and more weak on its periphery. A model of the chemical evolution of the disk of our Galaxy with variable gas accretion along the radius was constructed in [17]. It was concluded there that the characteristic time of accretion in which the local disk density increases significantly (by $e$ times) linearly increases along the radius: it is below 2 Gyr at the center, about $8 \mathrm{Gyr}$ near the Sun, and significantly exceeds the Hubble time on the far periphery (the disk only starts forming there). The characteristic times of times of largescale star formation change correspondingly. This concept was dubbed 'inside-out,' meaning that the (disk) galaxy was formed inside out. This concept has been confirmed by many observational facts.

One of the most spectacular confirmations was obtained by the ultraviolet space telescope GALEX (Galaxy Evolution Explorer), which obtained images of a large sample of nearby galaxies with good sensitivity and reasonable space resolution in the far $\left(\lambda_{e f f}=1516 \AA\right)$ and near $\left(\lambda_{e f f}=\right.$ $2267 \AA)$ ultraviolet spectrum [18]. It turned out that many disk galaxies have much larger sizes in the ultraviolet band than in the visible range [19]. What does this mean? Young massive stars are the main 'contributors of ultraviolet' in galaxies: they have high temperatures well exceeding 10,000 K, and hence the energy is mainly released in the ultraviolet range. After the completion of the galaxy survey by GALEX, it turned out that star formation occurs in external regions of galaxy disks, where there are almost no old stellar populations and nothing is visible in the optical range. This means that the disks are indeed built up, or more precisely, built over in the outer parts, in front of our very eyes.

Recently, an interesting study was carried out to test this scenario [20]. The evolution of ultraviolet (i.e., star-forming) disks was observed by directly comparing the sizes of galaxies at different redshifts. How can this be done? Because of a finite speed of light, the more distant the galaxy is, the earlier epoch is observed. For example, a galaxy at the redshift $z=0.5$ is seen five Gyr while the time delay for $z=1$ is eight Gyr. This means that using modern large-aperture telescopes, we can directly probe two thirds of the age of the Universe and can observe the evolution of galaxies galaxies during most of their lives. Due to the redshift, the proper ultraviolet emission from a remote galaxy can be observed in the optical range. The authors of [20] inspected the change in the characteristic shape of the disk surface brightness radial distribution with $z$ by adjusting the observed wavelength to the red- shift such that in the comoving frame of the galaxy, the measurements each time related to the same (ultraviolet) spectral range. They started from the GALEX galaxy survey carried out at $z \approx 0$. It turned out that indeed there is an evolution: at large redshifts, ultraviolet disks were observed to be more compact. At earlier times, star formation proceeded at the centers of the disks; has it now moved toward the periphery? This is exactly the evolution that is predicted by the inside-out scenario.

However, it turned out not to be so easy: when not only scales but also absolute levels of the ultraviolet surface brightness were compared, it was found that the peripheries of the disks appear almost identically at $z=1$ and $z=0$. The brightness profiles at $z=1$ are more compact or, in other words, have larger slopes due to star formation in their centers at $z=1$ occurring more intensively than at $z=0$, while no evolution of the star formation rate at the disk peripheries is observed. That is, the disks have not 'grown' in the last eight Gyr they have simply been completing the star formation at the center and continued it at the periphery. The inside-out scenario clearly needed to be improved.

Another problem of the disk formation theory is that no real gas reservoirs for accretion have been discovered to date, although it is difficult to doubt the reality of gas accretion on galactic disks. In addition, it is important that the external gas also has a low metal abundance. At some period, it was thought that during the collapse of a dark matter halo, the primordial gas gravitationally bound to it gradually heats up during virialization and is preserved for a long time as a hot X-ray halo around the galaxy. By gradually cooling down, the hot X-ray halo could provide a long steady accretion of the primordial gas onto the entire galactic disk. Such hot gas halos are observed in galaxy clusters, but none has been found so far around a nearby spiral galaxy (with the possible exception of massive bulge regions in early-type galaxies). Moreover, the detailed gasdynamic model showed that even if such halos exist, the known mechanisms of thermal instability of hot virialized (i.e., equilibrated) gas are unable to provide the required amount of cold gas clouds near galactic disks and the steady accretion over billions of years needed to build up a large-scale stellar disk [21].

For a long time, the outer gas source was 'nominated' to be high-velocity clouds of neutral hydrogen, which are actually observed outside the disk of our Galaxy. However, first, their number is too small to provide required accretion rates (at best they give 0.1-0.2 solar masses per year, which is one order of magnitude smaller than required to sustain the modern star formation rate). Second, when the heavy- element abundance was estimated by absorption lines formed in clouds serendipi- 
tously located along the line of sight, the chemical composition of the gas of high-velocity clouds was close to the solar one, and hence this was not the primordial gas. Presently, most high-velocity clouds of neutral hydrogen are thought to consist of gas ejected from the Galaxy by so-called galactic fountains gas outflows from active star formation regions, in which the gas is heated up by both stellar wind from massive stars and supernova explosions. This gas then cools to form clouds. However, first, this is not an 'addition' to the disk, but is originally a proper part of the disk, and second, the chemical composition is not the primordial one but is instead enriched by the products of nucleosynthesis.

In recent years, important changes have been occurring in the theory of galaxy formation. Hot virialized gas halos of young galaxies are now 'disfavored' as sources of matter for stellar disk formation; theoreticians doubt that the gas virialization occurs in most of the collapsing halos. The formation of disks and bulges of galaxies does not necessarily have to occur via the merging of smallsize subsystems only. A more and more important role in galaxy formation is probably played by cold filamentary gas flows directed to the inner part of a halo [22]. This is also a sort of flow accretion, but the accretion via gas streams that cannot occur on the entire disk and rather fuel its periphery. These cold streams pass without stopping through a hot gas halo and fall onto the disk. According to modern models [23], cold flows must dominate in low-mass (relative to the dark mass of clusters and groups of galaxies) halos at all redshifts starting from $z=5-6$. This means that there has been no effective gas accretion from outside onto the center of the disk at any stage of galactic evolution. Therefore, the inside-out galaxy building scenario in its classical formulation now conflicts with both observations and the cosmological theory. Clearly, the time for its cardinal revision is coming.

Our studies of galaxy disks are partially supported by the RFBR grants 07-02-00792 and 0702-00229.
[1] Makarov D, Karachentsev I Proc. Int. Astron. Union 3370 (2008); arXiv:0801.0043.

[2] Karachentsev I D, Makarov D I Astrofiz. Byull. 63320 (2008) [Astrophys. Bull. 63299 (2008)]; arXiv:0812.0689

[3] Bell E F, de Jong R S Astrophys. J. 550212 (2001)

[4] Zasov A V, Khoperskov A V, Tyurina N V Pis'ma Astron. Zh. 30653 (2004) [Astron. Lett. 30593 (2004)]

[5] Zasov A V et al. Pis'ma Astron. Zh. 28599 (2002) [Astron. Lett. 28527 (2002)]

[6] Bizyaev D, Mitronova S Astrophys. J. 7021567 (2009)

[7] Zasov A V, Khoperskov A V, Saburova A S, in preparation

[8] Dutton A A et al. Astrophys. J. 65427 (2007)

[9] Naim A et al. Mon. Not. R. Astron. Soc. 2741107 (1995)

[10] Paunzen E, Netopil M Mon. Not. R. Astron. Soc. 3711641 (2006)

[11] Wyse R F G Proc. Int. Astron. Union 4179 (2009);
arXiv:0809.4516

[12] Zemp M Mod. Phys. Lett. A 242291 (2009)

[13] Kazantzidis Set al. Astrophys. J. 7001896 (2009)

[14] Tosi M, astro-ph/0308463

[15] Feltzing S, Holmberg J, Hurley J R Astron. Astrophys. 377911 (2001)

[16] Jurgensen B R Astron. Astrophys. 363947 (2000)

[17] Chiappini C, Matteucci F, Gratton R Astrophys. J. 477765 (1997)

[18] Gil de Paz A et al. Astrophys. J. Suppl. 173185 (2007)

[19] Thilker D A et al. Astrophys. J. Suppl. 173538 (2007)

[20] Azzollini R, Beckman J E, Trujillo I Astron. Astrophys. 501119 (2009)

[21] Binney J, Nipoti C, Fraternali F Mon. Not. R. Astron. Soc. 3971804 (2009)

[22] Dekel A, Birnboim Y Mon. Not. R. Astron. Soc. 3682 (2006)

[23] Dekel A et al. Nature 457451 (2009) 